\title{
The conduct of Australian Indigenous primary health care research focusing on social and emotional wellbeing: a systematic review
}

\author{
Sara Farnbacha,b,i, Anne-Marie Eadesa,b,c , Josephine D Gwynn ${ }^{\mathrm{d}, e}$, \\ Nick Glozier ${ }^{f, g}$ and Maree L Hacketta,b,h
}

a The George Institute for Global Health, UNSW Sydney, Australia

b School of Medicine, University of Sydney, NSW, Australia

c Murdoch University, Perth, Western Australia

dPoche Centre for Indigenous Health, University of Sydney, NSW, Australia

e Faculty of Health Sciences, University of Sydney, NSW, Australia

${ }^{\dagger}$ Brain and Mind Centre, University of Sydney, NSW, Australia

g Central Clinical School, University of Sydney, NSW, Australia

${ }^{n}$ Faculty of Health and Wellbeing, University of Central Lancashire, Preston, UK

i Corresponding author: sfarnbach@georgeinstitute.org.au

\section{Article history}

Publication date: June 2018

Citation: Farnbach S, Eades AM, Gwynn JD, Glozier N, Hackett ML. The conduct of Australian Indigenous primary health care research focusing on social and emotional wellbeing: a systematic review. Public Health Res Pract. 2018;28(2):e27451704. https://doi.org/10.17061/phrp27451704

\section{Key points}

- Authors of Australian Indigenous primary health care research rarely report how their research addresses the national ethical guidelines, Values and ethics: guidelines for ethical conduct in Aboriginal and Torres Strait Islander health research (Values and ethics)

- Culturally sensitive approaches, developing relationships and involving community members appear to enable research, and uphold the principles in Values and ethics

- The academic community should focus on developing the Indigenous research workforce

- Authors should be encouraged to report actions and processes taken during research, to inform research planning and learning between research teams

\section{Abstract}

Objectives and importance of study: Values and ethics: guidelines for ethical conduct in Aboriginal and Torres Strait Islander health research (Values and ethics) describes key values that should underpin Aboriginal and Torres Strait Islander (Indigenous)-focused health research. It is unclear how research teams address this document in primary health care research. We systematically review the primary health care literature focusing on Indigenous social and emotional wellbeing (SEWB) to identify how Values and ethics and community preferences for standards of behaviour (local protocols) are addressed during research.

Study type: Systematic review in accordance with PRISMA Guidelines and MOOSE Guidelines for Meta-Analyses and Systematic Reviews of Observational Studies.

Methods: We searched four databases and one Indigenousspecific website for qualitative, quantitative and mixed-method studies published since Values and ethics was implemented (2003). Included studies were conducted in primary health care services, focused on Indigenous SEWB and were conducted by research teams. Using standard data extraction forms, we identified actions taken (reported by authors or identified by us) relating to Values and ethics and local protocols. 
Results: A total of 25 studies were included. Authors of two studies explicitly mentioned the Values and ethics document, but neither reported how their actions related to the document's values. In more than half the studies, we identified at least three actions relating to the values. Some actions related to multiple values, including use of culturally sensitive research processes and involving Indigenous representatives in the research team. Local protocols were rarely reported.

Conclusion: Addressing Values and ethics appears to improve research projects. The academic community should focus on culturally sensitive research processes, relationship building and developing the Indigenous research workforce, to facilitate acceptable research that affects health outcomes. For Values and ethics to achieve its full impact and to improve learning between research teams, authors should be encouraged to report how the principles are addressed during research, including barriers and enablers that are encountered.

\section{Introduction}

Primary health care research focusing on Aboriginal and Torres Strait Islander (Indigenous) peoples' needs is crucial to ensure evidence based and acceptable care is available. Perceptions that some past Indigenousfocused health research has provided minimal benefit, or excluded Indigenous people, have led to concerns surrounding Indigenous-focused research practices. ${ }^{1}$

To guide researchers, ethics committees and communities, Values and ethics: guidelines for ethical conduct in Aboriginal and Torres Strait Islander health research ${ }^{2}$ (Values and ethics) and its companion document ${ }^{3}$ were developed. For research involving Indigenous people, Values and ethics has the same status and authority as the National statement on ethical conduct in human research. ${ }^{4}$ Although some authors have described addressing the Values and ethics document during research ${ }^{5-8}$, its impact on research conduct is unclear. ${ }^{5,9}$ An evaluation of Values and ethics by the Lowitja Institute and the National Health and Medical Research Council (NHMRC) is under way. ${ }^{10}$

Primary health care services are considered the 'frontline' of the health system and are well positioned to identify and manage problems relating to social and emotional wellbeing (SEWB). The high rates of suicide and psychological distress among Indigenous people ${ }^{11}$ call for a particular focus on ensuring that SEWB care is effective, evidence based and acceptable. Research provides the framework to explore and assess SEWB care. Many Indigenous-focused primary health care services have programs or teams focusing on providing SEWB care. These services are often part of research teams involving primary health care staff, community members and externally located researchers, who collaborate to conduct SEWB research. ${ }^{12}$ Particular consideration of this research is needed because of the sensitive nature of research focused on SEWB and the challenges of implementing research in primary health care services.

Values and ethics identifies the following six values as key in underpinning research: reciprocity, respect, equality, responsibility, survival and protection, and spirit and integrity (see review protocol for definitions ${ }^{13}$ ). ${ }^{2}$ Values and ethics is an authoritative statement on Indigenous-focused health research. Other guidance documents include a practical guide for researchers ${ }^{14}$, a guideline for the ethical conduct of research ${ }^{15}$ and a document identifying important principles for Indigenous-focused health research. ${ }^{16}$ In previous work ${ }^{8}$, authors have drawn on the principles ${ }^{16}$ to examine the processes and procedures required to address its recommendations.

There is overlap across these documents ${ }^{2,14-16}$, with a common feature being to involve Indigenous representatives. However, there may be a lack of involvement, or reporting of involvement, of Indigenous people in research. This is demonstrated in a review of Indigenous child health research ${ }^{17}$ that identified involvement in only $28.6 \%$ of the 217 studies included.

Alongside Values and ethics, communities' preferences and priorities should be considered during research planning and conduct. Community preferences can be formally documented local protocols ${ }^{18}$, or undocumented standards of behaviour that research projects must adhere to within a community.

Using examples of Indigenous-focused SEWB primary health care research, we review and identify actions taken during research related to the application of Values and ethics and local protocols. Our previous review ${ }^{19}$ described the study designs, processes and main findings, and assessed the quality of the identified studies. 


\section{Methods}

The methods used in this review are previously published ${ }^{13,19}$, and are in accordance with PRISMA and MOOSE guidelines. This study is registered with PROSPERO (CRD42015024994). In brief, we searched Medline, Embase, CINAHL, Informit and HealthInfoNet. A date limit of January 2003 to February 2015 was applied to capture qualitative, quantitative or mixed-method studies conducted since the publication of Values and ethics. We included studies that were conducted in primary health care services, focused on Indigenous SEWB and that were conducted by research teams. We defined research teams as collaborations developed to conduct research that include primary health care staff or community members and researchers located outside the community. We included journal articles, reports and evaluations.

SEWB describes a strengths-based, holistic perspective of mental health that acknowledges social, historical and protective factors. ${ }^{20}$ In this review, we included SEWB, mental health, smoking or alcohol use, and depression and anxiety disorders. Primary health care services include Aboriginal medical services (AMSs), Aboriginal community controlled health services, and health services that provide primary health care or have general practitioners as staff members. Community refers to primary health care or AMS staff, patients, families or community members.

Data were extracted onto standard forms developed for this review. We identified when authors reported, or we identified actions taken relating to, the values detailed in Values and ethics using a previously developed list of potential actions ${ }^{13}$ and local protocols.

\section{Results}

Our search identified 2288 articles and projects. Following screening, 402 were found to be duplicates and 1491 articles were removed as they did not meet our inclusion criteria (described previously ${ }^{19}$ ). A total of 395 articles were reviewed by two reviewers, and 32 articles relating to 25 studies were included in the review (Supplementary Table 1 provides a ful reference list; available from: www.researchgate.net/ publication/317099307_FINAL_2017_05_25_Farnbach_ Systematic_Review_Supp_Tables). When two articles reported on one evaluation ${ }^{21,22}$ or project 23,24 and 25,26 , or articles appeared to report data collected from one set of surveys ${ }^{27,28}$, interview/focus group sessions 29,30 and 31,32 or questionnaires ${ }^{33,34}$, we included both articles and considered it as a single study.

The included studies focused on SEWB (nine) 25,26,31-40, alcohol misuse (five) $)^{27,28,41-44}$, smoking cessation (four) ${ }^{29,30,45-47}$ or dual diagnosis (SEWB and drug/ alcohol misuse; three).23,24,48,49 Two studies focused on depression. ${ }^{50,51}$ One focused on depression or anxiety ${ }^{52}$ and another on a mental health worker program. ${ }^{21,22}$

Three studies were part of the Australian Integrated Mental Health Initiative (AIMhi) ${ }^{25,26,36,37}$, a large research initiative aiming to improve outcomes for Indigenous clients of remote mental health services. The AIMhi 125,26, AIMhi $2^{36}$ and AIMhi $3^{37}$ studies have involved a research team known as the AIMhi Priority Driven Partnership which involved community-based and university-based researchers. ${ }^{38}$ Three studies were part of the Voices United for Harmony program, which developed and assessed a participatory singing program aimed at improving SEWB and physical health. ${ }^{33,34,39,40}$ Another three focused on alcohol screening and brief interventions in AMSs. ${ }^{41-43}$ One study that modified a psychological screening instrument ${ }^{50}$ was followed by another assessing its validity. ${ }^{51}$

\section{Use of Values and ethics and local protocols}

Authors explicitly mentioned Values and ethics in only two studies. ${ }^{44,47}$ In one ${ }^{47}$, authors identified their use of participatory action research methods as being in line with the document, and in the other study ${ }^{44}$, authors reported following Values and ethics during the research process. However, neither described specific actions relating to the values detailed in Values and ethics.

From the 25 studies, we identified 88 actions that related to (endorsed) the values in Values and ethics. Because each action could relate to more than one value, we identified a total of 146 endorsements of the values across all studies (Table 1). Several actions were identified in multiple studies (Table 2). Most common was acknowledging the contribution of primary health care staff $27-34,36,37,39,40,42,49,52$, services ${ }^{21,22,31-34,39,40,43,50}$, patients ${ }^{33,34,39,40,42,49}$, communities ${ }^{27,28}$, Indigenous organisations ${ }^{29,30}$ or community members ${ }^{52}$ in publications, or including staff as authors on publications. ${ }^{29,30,38,44-47,49-52}$ In seven studies, Indigenous representatives were involved with research teams. ${ }^{25,26,33,34,38-40,44,52}$ This endorsed five values. Authors of two studies ${ }^{35,43}$ reported visiting the community during research planning, with visits helping authors to understand the local context. ${ }^{43}$ Carey reports ${ }^{35}$ :

The researcher spent approximately 12 months travelling to the community to develop and build relationships ... these visits provided the principal researcher with an enhanced awareness of the functioning of the community, helped inform the design of the research, and promoted a greater understanding of the purpose of the research by members of the community.

The largest number of actions we identified from a single study was seven ( $n=3$ studies). 25,26,29,30,52 Some actions endorsed several values. For example, three studies used participatory action research methods $25,26,29,30,47$ demonstrating respect, equality, responsibility, and spirit and integrity. In one study ${ }^{33,34}$, 
authors modified the study to a nonrandomised design following community feedback, demonstrating reciprocity. This recognised the community's aspirations and demonstrated commitment to work within the spirit and integrity of the community.

Table 1. Percentage of studies with actions that endorsed values ${ }^{2}$ and the number of endorsements for each value

\begin{tabular}{lll}
\hline & $\begin{array}{l}\text { Studies with actions identified by } \\
\text { reviewers that endorsed each value } \\
(N=25 \text { studies), \% }(n)\end{array}$ & $\begin{array}{l}\text { Number of endorsements for each value } \\
(n=88 \text { actions; } n=146 \text { endorsements) }\end{array}$ \\
\hline Respect & $96(24)$ & 62 \\
Reciprocity & $60(15)$ & 20 \\
Survival and protection & $32(8)$ & 18 \\
Responsibility & $48(12)$ & 17 \\
Equality & $44(11)$ & 16 \\
Spirit and integrity & $52(13)$ & 13 \\
\hline
\end{tabular}

a 'Endorsements' are the number of times an action related to a value. Some actions endorsed (or related to) multiple values. 
Table 2. Summary of reported (by author) and identified (by reviewers) use of Values and ethics

\begin{tabular}{|c|c|c|c|c|c|c|}
\hline Action or process identified as addressing the values in Values and ethics (number of studies) & Reciprocity & Respect & Equality & Responsibility & $\begin{array}{l}\text { Survival and } \\
\text { protection }\end{array}$ & $\begin{array}{l}\text { Spirit and } \\
\text { integrity }\end{array}$ \\
\hline $\begin{array}{l}\text { Acknowledgement in the publication of primary health care staff }(n=11)^{27-34,36,37,39,40,42,49,52} \text {, services } \\
(n=8)^{21,22,31-34,39,40,43,50} \text {, patients }(n=5)^{33,34,39,40,42,49} \text {, communities }(n=1)^{27,28} \text {, Indigenous organisations } \\
(n=1)^{29,30} \text { or community members }(n=1)^{52}\end{array}$ & & $X$ & & & & \\
\hline $\begin{array}{l}\text { Publication authorship includes primary health care staff }(n=7)^{38,44,45(a), 46,47,49-51} \text { or Indigenous organisation } \\
\text { staff }(n=2)^{29,30,52}\end{array}$ & & $X$ & & & & \\
\hline $\begin{array}{l}\text { Research team involves Indigenous representatives: Community Elders }(n=3)^{33,34,39,40} \text {; primary health care } \\
\text { staff }(n=3)^{33,34,39,40 ;} \text { steering committee membership }(n=1)^{52} \text {; reference group membership }(n=1)^{44} \text {; as } \\
\text { investigators }(n=1)^{25,26} \text {; or families, carers and communities were involved }(n=1)^{38}\end{array}$ & & $X$ & $x$ & $X$ & $X$ & $X$ \\
\hline $\begin{array}{l}\text { Research interventions were informed by previous locally conducted studies }(n=2)^{41,43} \text { or feedback from } \\
\text { primary health care staff/patients/community }(n=3)^{25,26(c), 47,50}\end{array}$ & & $X$ & & & & \\
\hline Intervention developed within a collaborative framework $(n=1)^{46}$ & & $x$ & & & & \\
\hline Participants reimbursed for participation ( $n=5$ ) (voucher amount: $\$ 25^{45}, \$ 40^{27,28}, \$ 50^{35,49,52}$ ) & & $X$ & & & & \\
\hline Flexible interview location $(n=2)^{49,52}$, time $(n=1)^{45}$, or methods $(n=1)^{31,32}$ & $x$ & & & & & \\
\hline $\begin{array}{l}\text { Community identified need for research }(n=3) \text { (drug and alcohol services }{ }^{48} \text {, formal service evaluation }{ }^{35} \text {, or } \\
\text { alcohol screening and brief intervention }{ }^{42} \text { ) }\end{array}$ & $x$ & & & & & \\
\hline $\begin{array}{l}\text { Resources adapted for use by Indigenous people }(n=3) \text { (screening cut-off points }{ }^{27,28} \text {, depression } \\
\text { screening tool }{ }^{50} \text { or mental health strategy }{ }^{25,26} \text { ) }\end{array}$ & & $x$ & & & & \\
\hline $\begin{array}{l}\text { Participatory action research methods used }(n=1)^{25,26} \text { Used in combination with social-ecological } \\
\text { perspective }(n=1)^{29,30} \text { or yarning techniques }(n=1)^{47}\end{array}$ & & $x$ & $x$ & $x$ & & $x$ \\
\hline Research approved by community research governance committee $(n=4)^{25,26,31,32,44,45}$ & & $X$ & & & & \\
\hline Consultation informed resources and training materials $(n=1)^{23,24}$ or study instruments $(n=1)^{27,28(d)}$ & & $X$ & & & & \\
\hline Study planning and implementation driven by primary health care staff $(n=2)^{38,45(e)}$ & & $X$ & & & $X$ & \\
\hline $\begin{array}{l}\text { Regular visits during planning to understand local processes/context }(n=1)^{43} \text { or to develop research } \\
\text { methods }(n=1)^{35}\end{array}$ & & & & & & $x$ \\
\hline Informed consent involved two-step process $(n=1)^{35}$ or written, pictorial and translation options $(n=1)^{25,26}$ & & & & $x$ & & \\
\hline 'Two-way learning' processes used $(n=2)^{21,22,29,30}$ & $x$ & $x$ & $X$ & $x$ & $x$ & $x$ \\
\hline
\end{tabular}




\begin{tabular}{|c|c|c|c|c|c|c|}
\hline Action or process identified as addressing the values in Values and ethics (number of studies) & Reciprocity & Respect & Equality & Responsibility & $\begin{array}{l}\text { Survival and } \\
\text { protection }\end{array}$ & $\begin{array}{l}\text { Spirit anc } \\
\text { integrity }\end{array}$ \\
\hline Action plan developed to implement research findings $(n=1)^{48}$ & $x$ & & & & & \\
\hline Resources will remain with the community $(n=1)^{23,24}$ & $x$ & & & & & \\
\hline Phenomenological research methods used $(n=1)^{45}$ & & $x$ & & & $x$ & \\
\hline Interviews conducted by Indigenous community member $(n=1)^{45}$ & & $x$ & & & & $x$ \\
\hline $\begin{array}{l}\text { Chief Executive Officer at primary health care service approved publications or results before release } \\
(n=1)^{27,28}\end{array}$ & & & & $x$ & & \\
\hline $\begin{array}{l}\text { Intention to provide information to other communities by identifying processes instead of programs in } \\
\text { evaluation }(n=1)^{35}\end{array}$ & $x$ & & & & & \\
\hline Study design developed in conjunction with the research governance committee (health board) $(n=1)^{35}$ & & & $x$ & $X$ & & $x$ \\
\hline $\begin{array}{l}\text { Project underpinned by six Iga Warta principles for Aboriginal health projects (prevention, coordination, } \\
\text { sustainability, social determinants of health, sensitivity to Indigenous notions of time and space, and } \\
\text { community and family) }(n=1)^{29,30}\end{array}$ & & $x$ & & & & $x$ \\
\hline Focus on knowledge translation, and findings provided to stakeholders $(n=1)^{29,30}$ & $x$ & & & & & \\
\hline Cultural mentorship of researchers by respected Elder $(n=1)^{29,30}$ & & & & & $x$ & \\
\hline $\begin{array}{l}\text { Visits by researcher during research according to Aboriginal medical service needs and preferences } \\
(n=1)^{42}\end{array}$ & $x$ & & & & & \\
\hline Study proposed by the Indigenous organisation $(n=1)^{52}$ & & & $x$ & & & \\
\hline $\begin{array}{l}\text { Regular feedback provided to stakeholders. Steering committee (including community representatives) } \\
\text { provided feedback on findings }(n=1)^{52}\end{array}$ & & & $x$ & $x$ & & \\
\hline Authors did not publish some findings to protect participant confidentiality $(n=1)^{49}$ & & & & & $x$ & \\
\hline Focus on providing training to primary health care staff $(n=1)^{44}$ & $x$ & & & & & \\
\hline Research underpinned by empowerment principles $(n=1)^{38}$ & $x$ & & & & & \\
\hline Study modified to nonrandomised design following community feedback $(n=1)^{33,34}$ & $x$ & & & & & $x$ \\
\hline
\end{tabular}


Involving Indigenous community representatives in key positions incorporated Indigenous knowledge and experience into research (respect). It was common to involve Community Elders ${ }^{33,34,39,40}$, primary health care staff ${ }^{33,34,39,40}$, families, carers and communities ${ }^{38}$, or any of these as members of a steering committe ${ }^{52}$, reference group ${ }^{44}$ or as investigators. ${ }^{25,26}$ Consultations to inform resources ${ }^{23,24}$ or study instrument ${ }^{27,28}$ development were reported twice. Acknowledging the contribution of participants (respect) by providing shopping or food vouchers was reported in five studies. ${ }^{27,28,35,45,49,52}$ Willingness to modify research according to a community's values and aspirations through flexible research processes (reciprocity) was also common. This included flexible interview times, locations or methods ${ }^{31,32,41,45,49,52}$; multiple visits during planning ${ }^{43}$; or modifying study design following community feedback. ${ }^{33,34}$

Authors of three studies reported using 'two-way learning' principles, which demonstrated equality; survival and protection (efforts to reduce the threat to cultural distinctiveness); and respect (incorporating Indigenous knowledge). This included the Aboriginal Mental Health Worker Program evaluation ${ }^{21,22}$, AIMhi $1^{25,26}$ and a smoking cessation study with Aboriginal health workers. ${ }^{29,30}$ The smoking cessation study ${ }^{29,30}$ also used participatory action research methods, had a cultural mentor to advise researchers and was underpinned by Iga Warta principles, a set of guiding principles for community participation and service delivery in Indigenous communities. These actions also demonstrated survival and protection, spirit and integrity, and respect.

Some of the other actions relating to survival and protection included involving families, carers or community representatives in the research team. ${ }^{25,26,33,34,38-}$ 40,44,52 In addition to using participatory action research methods, Indigenous researchers in AIMhi 1 were investigators ${ }^{25,26}$, demonstrating efforts to sustain equality and reduce the threat to cultural distinctiveness (survival and protection). Authors of one study decided not to publish some findings to protect the confidentiality of participants. ${ }^{49}$

No authors reported compliance with documented local protocols; however, a respected Elder provided cultural mentorship to the research team in one study. ${ }^{29,30}$ This suggests consideration of locally acceptable standards of behaviour.

\section{Discussion}

Our results show that reporting of how research addresses Values and ethics ${ }^{2}$ is lacking. This suggests that authors may find it difficult to put value statements into practice, a lack of focus on or knowledge of the document, perceptions that reporting observance is unimportant, or that it is not perceived as useful. Reporting incorporation of local protocols is also lacking.
Some actions may be underreported because academic journals often impose word limits, restricting reporting of nonmandatory elements of research.

Many of the actions identified that related to Values and ethics were reported as enablers to conducting the research. This suggests that awareness and consideration of the document may improve research implementation. For example, relationships are a key component of Values and ethics, and authors of three studies $29,30,35,38$ reported strong relationships as an enabler. These relationships were fostered through:

- Involving community organisations and/or key community representatives; this endorses reciprocity, respect, equality, responsibility, spirit and integrity, and survival and protection

- Visiting communities before starting the research; this endorses reciprocity, and spirit and integrity

- A focus on empowerment principles, which endorses reciprocity.

Actions that related to (or endorsed) multiple values used culturally sensitive research processes, rather than one-off actions incorporated into traditional evidence based research methods. These included two-way learning 21,22,25,26,29,30, yarning ${ }^{47}$, participatory action research methods ${ }^{25,26,29,30,47}$ and Iga Warta principles. ${ }^{29,30}$ In one study ${ }^{33,34}$, the design was changed to a nonrandomised design following community feedback, demonstrating the challenges associated with aligning community preferences with what is usually considered scientifically rigorous research.

Involving Indigenous community members in research roles was common, although recruiting Indigenous staff was cited as a barrier to research implementation in one study. ${ }^{29,30} \mathrm{~A}$ focus on developing the Indigenous research workforce may address challenges with staffing and participation by facilitating research with greater community endorsement.

There are a few examples of others ${ }^{5-8}$ who have documented research according to Values and ethics. Interestingly, these examples identify relationships and partnerships as important facilitators to their research, echoing the processes identified in this review.

We suggest that research teams consider the actions identified that relate to Values and ethics. These include culturally sensitive approaches, a focus on relationship building and involving community members. Where appropriate, we recommend that reporting of research includes documentation of actions, experiences and community perspectives, and how these relate to Values and ethics. This will support shared learning between research teams and help clarify the effectiveness, cost and time required to implement research.

This review suggests that it is difficult to understand how Values and ethics is put into practice. Identifying and using culturally appropriate research methods requires commitment from research teams and the academic community. Academic publications may need to increase word limits so research teams can report 
research processes from all perspectives. This will provide information on the role and potential for Values and ethics to support high-quality, community-accepted research when primary health care services and external researchers collaborate.

We have considered the values outlined in Values and ethics throughout this review. The second reviewer and author is an Aboriginal researcher and has been involved since this review's inception, including during protocol development, data extraction and analysis. This manuscript has been reviewed by the Aboriginal Health \& Medical Research Council of New South Wales. This review responds to ongoing calls for improved research practices of Indigenous-focused research. We hope it provides useful information to Indigenous communities, primary health care services and research teams.

This review is limited to the information reported by authors. Additional actions may have been completed but not documented. Determining cultural appropriateness and community perspectives from the literature is challenging. We have identified where this is reported, but this may not fully identify the extent to which this has occurred. Indigenous communities are diverse, and an appropriate action in one community may not be suitable for another community.

\section{Conclusion}

Despite a lack of reporting, it appears that incorporation of the principles in Values and ethics improves research implementation. A focus on relationships and involving community members facilitates research in accordance with the Values and ethics document. Research teams should incorporate flexible, culturally sensitive designs to inform localised interventions, and focus on developing Indigenous researchers. Comprehensive reporting of how research is conducted should be encouraged to ensure community-level benefit and learning between research teams. The evaluation by the Lowitja Institute and the NHMRC will provide further information on the future of Values and ethics.

\section{Acknowledgements}

We acknowledge staff at the University of Sydney Medical Library for their help developing the search strategy for this review. AE received a National Health Research Scholarship (APP1056434). MH received a National Heart Foundation Future Leader Fellowship (100034). These funding bodies had no role in the conduct or reporting of this review.

\section{Peer review and provenance}

Externally peer reviewed, not commissioned

\section{Competing interests}

None declared

\section{Author contributions}

SF led this review. AE was second author and second reviewer. NG and JG contributed to the methods and discussion. MH supervises SF and AE. All authors contributed to the final manuscript.

\section{References}

1. Hayman N, Papaarangi M, King M. Improving health outcomes for Indigenous peoples: what are the challenges? Cochrane Database Syst Rev. 2015;(8):ED000104.

2. National Health and Medical Research Council. Values and ethics: guidelines for ethical conduct in Aboriginal and Torres Strait Islander health research. Canberra: Commonwealth of Australia; 2003 [cited 2014 Sep 1]. Available from: www.nhmrc.gov.au/_files_nhmrc/ publications/attachments/e52.pdf

3. National Health and Medical Research Council. Keeping research on track: a guide for Aboriginal and Torres Strait Islander peoples about health research ethics. Canberra: Australian Government; 2005 [cited 2017 Nov 1]. Available from: www.nhmrc.gov.au/_files_nhmrc/ publications/attachments/e65.pdf

4. National Health and Medical Research Council. National statement on ethical conduct in human research 2007 (updated May 2015). Canberra: Commonwealth of Australia; 2015 [cited 2017 Nov 1]. Available from: www. nhmrc.gov.au/_files_nhmrc/publications/attachments/ e72_national_statement_may_2015_150514_a.pdf

5. Bainbridge R, Tsey K, Brown C, McCalman J, CadetJames Y, Margolis S, Ypinazar V. Coming to an ethics of research practice in a remote Aboriginal Australian community. Contemp Nurse. 2013;46(1):18-27.

6. Knight J, Comino E, Harris E, Jackson-Pulver L. Indigenous research: a commitment to walking the talk. The Gudaga Study - an Australian case study. J Bioeth Inq. 2009;6(4):467-76.

7. Isaacs AN, Pepper H, Pyett P, Gruis HA, WaplesCrowe $P$, Oakley-Browne MA. 'What you do is important but how you do it is more important': engaging Indigenous men in rural mental health services research. Qualitative Research Journal. 2011;11(1):51.

8. Gwynn J, Lock M, Turner N, Dennison R, Coleman C, Kelly B, Wiggers J. Aboriginal and Torres Strait Islander community governance of health research: turning principles into practice. Aust J Rural Health. 2015;23(4):235-42. 
9. Dunbar T, Scrimgeour M. Ethics in Indigenous research - connecting with community. J Bioeth Inq. 2006;3(3):179-85.

10. Australian Institute of Aboriginal and Torres Strait Islander Studies, The Lowitja Institute. Evaluation of the National Health and Medical Research Council documents: Values and ethics: guidelines for ethical conduct in Aboriginal and Torres Strait Islander health research 2003 and Keeping research on track: a guide for Aboriginal and Torres Strait Islander peoples about health research ethics 2005. Canberra: National Health and Medical Research Council; 2013 [cited 2017 Nov 1]. Available from: www.nhmrc.gov.au/_files_nhmrc/file/health_ethics/ human/issues/nhmrc_evaluation_values_ethics_research_ on_track_150513.pdf

11. Australian Bureau of Statistics. Australian Aboriginal and Torres Strait Islander health survey: first results, Australia, 2012-13. Psychological distress. Canberra: ABS; 2013 [cited 2014 Sep 23]. Available from: www.abs.gov.au/ ausstats/abs@//9F3C9BDE98B3C5F1CA257C2F0014572 1?opendocument

12. Mc Loughlin F, Hadgraft N, Atkinson D, Marley J. Aboriginal health research in the remote Kimberley: an exploration of perceptions, attitudes and concerns of stakeholders. BMC Health Serv Res. 2014;14(1):517-28.

13. Farnbach S, Eades AM, Hackett M. Australian Aboriginal and Torres Strait Islander-focused primary healthcare social and emotional wellbeing research: a systematic review protocol. Syst Rev. 2015;4(189).

14. Laycock A, Walker D, Harrison N, Brands J. Researching Indigenous health: a practical guide for researchers. Melbourne: The Lowitja Institute; 2011 [cited 2017 Nov 1]. Available from: www.lowitja.org.au/researchers-guide

15. Australian Institute of Aboriginal and Torres Strait Islander Studies. Guidelines for ethical research in Australian Indigenous studies. Canberra: AIATSIS; 2012 [cited 2017 Nov 1]. Available from: aiatsis.gov.au/sites/default/ files/docs/research-and-guides/ethics/GERAIS.pdf

16. Jamieson L, Paradies Y, Eades S, Chong A, MapleBrown L, Morris $P$, et al. Ten principles relevant to health research among Indigenous Australian populations. Med J Aust. 2012;197(1):16-8.

17. Priest N, Mackean T, Waters E, Davis E, Riggs E. Indigenous child health research: a critical analysis of Australian studies. Aust N Z J Public Health. 2009;33(1):55-63.

18. Hurley A. Respect, acknowledge, listen: practical protocols for working with the Indigenous community of Western Sydney 2003. Sydney: Community and Cultural Development NSW Ltd; 2003 [cited 2017 Nov 1]. Available from: www.wipo.int/export/sites/www/tk/en/ databases/creative_heritage/docs/ccdnsw_indig_ westsydney.pdf

19. Farnbach S, Eades AM, Fernando J, Gwynn J, Glozier N, Hackett $M$. The quality of Australian Indigenous primary health care research focusing on social and emotional wellbeing: a systematic review. Public Health Res Pract. 2017;27(4):e27341700.
20. Kelly K, Dudgeon P, Gee G, Glaskin B. Living on the edge: social and emotional wellbeing and risk and protective factors for serious psychological distress among Aboriginal and Torres Strait Islander people. Darwin: Australian Indigenous Psychologists Association and Cooperative Research Centre for Aboriginal Health; 2009. Available from: www.indigenouspsychology.com. au/Assets/Files/AIPA-Living-on-the-Edge-web.pdf

21. Harris A, Robinson G. The Aboriginal Mental Health Worker Program: the challenge of supporting Aboriginal involvement in mental health care in the remote community context. Australian e-Journal for the Advancement of Mental Health. 2007;6(1):11.

22. Robinson G, Harris A. Aboriginal Mental Health Worker Program: final evaluation report. Darwin: School for Social and Policy Research, Institute of Advanced Studies, Charles Darwin University; 2004 [cited 2017 Jun 2]. Available from: nceta.flinders.edu.au/ files/6813/5787/2431/Abor_mh_worker_program_final.pdf

23. Bakos K, Victorian Dual Diagnosis Education and Training Unit. Indigenous dual diagnosis training project stage 1: the consultation report. Melbourne: Victorian Dual Diagnosis Education and Training Unit; 2008. Copy available from author.

24. Bakos K, Logan G. Indigenous dual diagnosis training project stage 3: training evaluation report. Melbourne: Victorian Dual Diagnosis Initiative, St Vincent's Health Australia. Copy available from author.

25. Nagel T, Thompson C, Robinson G, Trauer T, Condon J. Two-way approaches to Indigenous mental health literacy. Aust J Prim Health. 2009;15(1):50-5.

26. Nagel T, Robinson G, Condon J, Trauer T. Approach to treatment of mental illness and substance dependence in remote Indigenous communities: results of a mixed methods study. Aust J Rural Health. 2009;17(4):174-82.

27. Calabria B, Clifford A, Shakeshaft A, Allan J, Bliss D, Doran $\mathrm{C}$. The acceptability to Aboriginal Australians of a family-based intervention to reduce alcohol-related harms. Drug Alcohol Rev. 2013;32(3):328-32.

28. Calabria B, Clifford A, Shakeshaft A, Conigrave K, Simpson L, Bliss D, et al. Identifying Aboriginal-specific AUDIT-C and AUDIT-3 cutoff scores for at-risk, highrisk, and likely dependent drinkers using measures of agreement with the 10-item Alcohol Use Disorders Identification Test. Addict Sci Clin Pract. 2014;9:17.

29. Dawson A, Cargo M, Stewart H, Chong A, Daniel M. Aboriginal health workers experience multilevel barriers to quitting smoking: a qualitative study. Int J Equity Health. 2012;11:27.

30. Dawson AP, Cargo M, Stewart H, Chong A, Daniel M. "I know it's bad for me and yet I do it": exploring the factors that perpetuate smoking in Aboriginal health workers - a qualitative study. BMC Health Serv Res. 2012;12:102.

31. Williamson A, Redman S, Dadds M, Daniels J, D'Este C, Raphael B, et al. Acceptability of an emotional and behavioural screening tool for children in Aboriginal Community Controlled Health Services in urban NSW. Aust N Z J Psychiatry. 2010;44(10):894-900. 
32. Raphael B, Redman S, Daniels J, Eades S, Mayers N Emerging themes in Aboriginal child and adolescent mental health: findings from a qualitative study in Sydney, New South Wales. Med J Aust. 2010;192(10):603-5.

33. Sun J, Buys N. Effectiveness of a participative community singing program to improve health behaviors and increase physical activity in Australian Aboriginal and Torres Strait Islander people. Int J Disabil Hum Dev. 2013;12(3):297-304

34. Sun J, Buys N. Effects of community singing program on mental health outcomes of Australian Aboriginal and Torres Strait Islander people: a meditative approach. Am J Health Promot. 2016;30(4):259-63.

35. Carey TA. A qualitative study of a social and emotional well-being service for a remote Indigenous Australian community: implications for access, effectiveness, and sustainability. BMC Health Serv Res. 2013;13:80.

36. Nagel T. The need for relapse prevention strategies in Top End remote Indigenous mental health. Australian e-Journal for the Advancement of Mental Health. 2006;5(1):1-5.

37. Dingwall K, Puszka S, Sweet M, Nagel T. "Like drawing into sand": acceptability, feasibility, and appropriateness of a new e-mental health resource for service providers working with Aboriginal and Torres Strait Islander people. Aust Psychologist. 2012;50(1):60-9

38. Haswell-Elkins M, Reilly L, Fagan R, Ypinazar V, Hunter $\mathrm{E}$, Tsey $\mathrm{K}$, et al. Listening, sharing understanding and facilitating consumer, family and community empowerment through a priority driven partnership in Far North Queensland. Australas Psychiatry. 2009;17 Suppl(2009):S54-8.

39. Sun J, Buys N. Participatory community singing program to enhance quality of life and social and emotional wellbeing in Aboriginal and Torres Strait Islander Australians with chronic diseases. Int Journal Disabil Hum Dev. 2013;12(3):317-23.

40. Sun J, Buys N. Improving Aboriginal and Torres Strait Islander Australians' well-being using participatory community singing approach. Int J Disabil Hum Dev. 2013;12(3):305-16.

41. Clifford A, Shakeshaft A, Deans C. Training and tailored outreach support to improve alcohol screening and brief intervention in Aboriginal Community Controlled Health Services. Drug Alcohol Rev. 2013;32(1):72-9.

42. Clifford A, Shakeshaft A. Evidence-based alcohol screening and brief intervention in Aboriginal Community Controlled Health Services: experiences of health-care providers. Drug Alcohol Rev. 2011;30(1):55-62.
43. Clifford A, Shakeshaft A, Deans C. How and when healthcare practitioners in Aboriginal Community Controlled Health Services deliver alcohol screening and brief intervention, and why they don't: a qualitative study. Drug Alcohol Rev. 2012;31(1):13-9.

44. Lovett R, Dance P, Guthrie J, Brown R, Tongs J. Walan Girri: developing a culturally mediated case management model for problematic alcohol use among urban Indigenous people. Aust Health Rev. 2014;38(4):440-6.

45. Bond C, Brough M, Spurling G, Hayman N. 'It had to be my choice' Indigenous smoking cessation and negotiations of risk, resistance and resilience. Health Risk Soc. 2012;14(6):565-81.

46. DiGiacomo M, Davidson PM, Davison J, Moore L, Abbott P. Stressful life events, resources, and access: key considerations in quitting smoking at an Aboriginal Medical Service. Aust N Z J Public Health. 2007;31(2):174-6.

47. Fletcher G, Fredericks B, Adams K, Finlay S, Andy S, Briggs L, Hall R. Having a yarn about smoking: using action research to develop a 'no smoking' policy within an Aboriginal Health Organisation. Health Policy. 2011;103(1):92-7.

48. Allan J. Engaging primary health care workers in drug and alcohol and mental health interventions: challenges for service delivery in rural and remote Australia. Aust J Prim Health. 2010;16(4):311-8.

49. Lee KS, Harrison K, Mills K, Conigrave KM. Needs of Aboriginal Australian women with comorbid mental and alcohol and other drug use disorders. Drug Alcohol Rev. 2014;33(5):473-81.

50. Esler D, Johnston F, Thomas D. The acceptability of a depression screening tool in an urban, Aboriginal community-controlled health service. Aust N Z J Public Health. 2007;31(3):259-63.

51. Esler D, Johnston F, Thomas D, Davis B. The validity of a depression screening tool modified for use with Aboriginal and Torres Strait Islander people. Aust N Z J Public Health. 2008;32(4):317-21.

52. Higgins R, Murphy B, Jobling K. Improving the wellbeing of Melbourne-based Aboriginal people with chronic disease and experiencing depression, anxiety or a related mental health disorder: a Wurundjeri community driven initiative. Melbourne: Heart Research Institute, The University of Melbourne, Wurundjeri Tribe; 2013 [cited 2017 Jun 2]. Available from: www. beyondblue.org au/docs/default-source/research-project-files/bw0165. pdf?sfvrsn=2

\section{Copyright: (cc)}

(C) 2018 Farnbach et al. This article is licensed under the Creative Commons Attribution-NonCommercial-ShareAlike 4.0 International Licence, which allows others to redistribute, adapt and share this work non-commercially provided they attribute the work and any adapted version of it is distributed under the same Creative Commons licence terms. See: www.creativecommons.org/licenses/by-nc-sa/4.0/ 